\title{
Kısıtlamasız $\mathbf{k}$ - Fibonacci ve $\mathbf{k}$ - Lucas genelleştirilmiş kuaterniyonları
}

\author{
Unrestricted $k$ - Fibonacci and $k$ - Lucas generalized quaternions \\ Göksal BİLGICI*1,a \\ ${ }^{1}$ Kastamonu Üniversitesi, Eğitim Fakültesi, Matematik Eğitimi Anabilim Dall, 37200, Kastamonu
}

• Geliş tarihi / Received: 23.04.2020 • • Düzeltilerek geliş tarihi / Received in revised form: 14.12.2020 • Kabul tarihi / Accepted: 23.12 .2020

\begin{abstract}
Öz
Literatürde Fibonacci ve Lucas sayılarının birçok genelleştirilmesi bulunmaktadır. Bu genelleştirmelere bir örnek olarak $\mathrm{k}$ - Fibonacci ve $\mathrm{k}$ - Lucas sayıları verilebilir. Bu çalışmada kısıtlamasız $\mathrm{k}$ - Fibonacci ve $\mathrm{k}$ - Lucas genelleştirilmiş kuaterniyonları tanımlanmıştır. Kısıtlamasız kelimesinden, kuaterniyonların sıralı tabanındaki versörlerin katsayılarının keyfi $\mathrm{k}$ - Fibonacci ve $\mathrm{k}$ - Lucas sayısı olarak atanabilmesi kastedilmektedir. Bu doğrultuda, kısıtlamasız k - Fibonacci ve $\mathrm{k}$ - Lucas genelleştirilmiş kuaterniyonlarının üreteç fonksiyonları ve Binet formülleri elde edildikten sonra, bilinen bazı özdeşliklerin genelleştirmeleri verilmiştir.
\end{abstract}

Anahtar kelimeler: Genelleştirilmiş kuaterniyonlar, k - Fibonacci sayıları, k -Lucas sayıları

\begin{abstract}
There are many generalizations of Fibonacci and Lucas numbers. One of them is $k$ - Fibonacci and $k$ - Lucas numbers. In this study, we introduce unrestricted $k$ - Fibonacci and $k$-Lucas generalized quaternions. The word "unrestricted" means that we can determine the coefficients of the versors of the basis of the quaternions arbitrarily. In this manner, we give generating functions and Binet formulas for the unrestricted $k$ - Fibonacci and $k$-Lucas generalized quaternions and obtain generalizations of some well - known identities.
\end{abstract}

Keywords: Generalized quaternions, $k$ - Fibonacci numbers, $k$ - Lucas numbers

*a Göksal BİLGİĊ; gbilgici@kastamonu.edu.tr, Tel: (0366) 28033 38, orcid.org/0000-0001-9964-5578 


\section{Giriş}

Fibonacci ve Lucas dizilerinin tamsayı dizileri arasında en şöhretli diziler oldukları söylenebilir. Fibonacci ve Lucas sayıları sirasıyla;

$F_{0}=0, F_{1}=1, F_{n}=F_{n-1}+F_{n-2} \quad(n \geq 0)$ ve $L_{0}=2, L_{1}=1, L_{n}=L_{n-1}+L_{n-2} \quad(n \geq 0)$

bağıntıları ile tanımlanırlar. Fibonacci sayılarına, bu sayılar Leonardo Fibonacci’nin "Liber Abaci” isimli kitabının sonunda bırakmış olduğu tavşan probleminin çözümü olması nedeniyle bu isim verilmiştir. Bu sayılar günlük hayatımızın birçok yerinde karşımıza çıkabilirler. Örnek olarak doğa, müzik ve finansal piyasalar verilebilir (Koshy, 2001).

Fibonacci ve Lucas dizilerine benzer olarak Pell ve Pell - Lucas sayıları sırasıyla;

$$
P_{0}=0, P_{1}=1, P_{n}=2 P_{n-1}+P_{n-2} \quad(n \geq 0) \text { ve } Q_{0}=1, Q_{1}=1, Q_{n}=2 Q_{n-1}+Q_{n-2} \quad(n \geq 0)
$$

bağıntıları vasıtasıyla tanımlanırlar. Pell sayıları ilk olarak bazı Diophantine denklemlerinin çözümü olarak karşımıza çıkar (Koshy, 2014). $\left\{F_{n}\right\},\left\{L_{n}\right\},\left\{P_{n}\right\}$ ve $\left\{Q_{n}\right\}$ dizileri için üreteç fonksiyonları sırasıyla

$$
\sum_{n=0}^{\infty} F_{n} x^{n}=\frac{x}{1-x-x^{2}}, \sum_{n=0}^{\infty} L_{n} x^{n}=\frac{2-x}{1-x-x^{2}}, \sum_{n=0}^{\infty} P_{n} x^{n}=\frac{x}{1-2 x-x^{2}} \text { ve } \sum_{n=0}^{\infty} Q_{n} x^{n}=\frac{2-x}{1-2 x-x^{2}}
$$

şeklindedir. Fibonacci ve Lucas sayıları için Binet formülleri

$$
F_{n}=\frac{\alpha^{n}-\beta^{n}}{\alpha-\beta} \text { ve } L_{n}=\alpha^{n}+\beta^{n}
$$

dir. Burada $\alpha=\frac{1+\sqrt{5}}{2}$ ve $\beta=\frac{1-\sqrt{5}}{2}$ sayıları $x^{2}-x-1=0$ karakteristik denkleminin kökleridir. Pozitif kök ${ }_{\alpha}$ altın oran olarak bilinir ve Fibonacci sayıları teorisinde çok önemli bir rol oynamaktadır. Pell ve Pell - Lucas sayılarının Binet formülleri ise sırasıyla;

$$
P_{n}=\frac{\gamma^{n}-\delta^{n}}{\gamma-\delta} \text { ve } Q_{n}=\frac{\gamma^{n}+\delta^{n}}{2}
$$

şeklindedir. Burada $\gamma=1+\sqrt{2}$ ve $\delta=1-\sqrt{2}$ sayıları $x^{2}-2 x-1=0$ karakteristik denkleminin kökleridir. Pozitif kök $\gamma$, metalik oranlar arasında gümüş oran olarak bilinir ve altın orana benzer bir rolü vardır. Fibonacci ve Lucas dizilerinin birçok ilginç özelliği bulunmaktadır. Bu özellikleri başka dizilere genişletmek amacıyla birçok genelleştirmenin yapıldığı görülmektedir. Bu genelleştirmeler için iki temel yöntem göze çarpmaktadır. Birinci yöntem başlangıç koşullarını koruyup rekörsif bağıntıyı değiştirmek iken ikinci yöntem ise rekörsif bağıntıyı koruyup başlangıç koşullarını değiştirmektir.

Birinci yönteme bir örnek Falcon ve Plaza (Falcon ve Plaza, 2007) tarafindan tanımlanan $k$ - Fibonacci sayılarıdır. $k$ bir pozitif reel sayı olmak üzere $k-$ Fibonacci sayıları

$$
F_{k, n}=k F_{k, n-1}+F_{k, n-2}
$$

rekörsif formülü ile tanımlanırken başlangıç koşulları $F_{k, 0}=0$ ve $F_{k, 1}=1$ şeklindedir. $k$ - Lucas sayıları ise Falcon (Falcon, 2011) tarafindan aşağıdaki rekörsif formül ile tanımlanmıştır

$$
L_{k, n}=k L_{k, n-1}+L_{k, n-2} .
$$


Başlangıç koşulları $L_{k, 0}=2$ ve $L_{k, 1}=k$ şeklindedir. $k=1$ için $k$-Fibonacci ve $k$-Lucas sayıları sırasıyla Fibonacci ve Lucas sayılarını verirken, $k=2$ için ise Pell ve Pell - Lucas sayılarını verirler. $\left\{F_{k, n}\right\}$ ve $\left\{L_{k, n}\right\}$ dizileri için üreteç fonksiyonları sırasıyla;

$\sum_{n=0}^{\infty} F_{k, n} x^{n}=\frac{x}{1-k x-x^{2}}$ ve $\sum_{n=0}^{\infty} L k_{k, n} x^{n}=\frac{2-x}{1-k x-x^{2}}$

iken $k$ - Fibonacci ve $k$ - Lucas sayıları için Binet formülleri sırasıyla;

$$
F_{k, n}=\frac{\chi^{n}-\mu^{n}}{\chi-\mu} \text { ve } L_{k, n}=\chi^{n}+\mu^{n}
$$

şeklindedir. Burada $\chi=\frac{k+\sqrt{k^{2}+4}}{2}$ ve $\mu=\frac{k-\sqrt{k^{2}+4}}{2}$ sayıları $x^{2}-k x-1=0$ karakteristik denkleminin kökleridir.

Kuaterniyonlar, Sir W.R. Hamilton tarafından 1843 yılında keşfedilmiştir (Hamilton, 1853). Sonrasında ise başka kuaterniyonların tanımlandığı görülmektedir. $\mathrm{Bu}$ çalışmada iki parametreli genelleştirilmiş kuaterniyonlar çalışılacaktır. $\sigma$ ve $\zeta$ reel sayılar olmak üzere genelleştirilmiş kuaterniyonların kümesi

$K=\left\{a+b e_{1}+c e_{2}+d e_{3}: a, b, c, d \in \mathbb{R}\right\}$

olarak tanımlanır. $\left\{1, e_{1}, e_{2}, e_{3}\right\}$ taban elemanlarının çarpımı aşağıdaki tablodaki gibidir:

Tablo 1. $\left\{1, e_{1}, e_{2}, e_{3}\right\}$ taban elemanlarının çarpımı

\begin{tabular}{c|cccc}
. & 1 & $e_{1}$ & $e_{2}$ & $e_{3}$ \\
\hline 1 & 1 & $e_{1}$ & $e_{2}$ & $e_{3}$ \\
$e_{1}$ & $e_{1}$ & $-\sigma$ & $e_{3}$ & $-\sigma e_{2}$ \\
$e_{2}$ & $e_{2}$ & $-e_{3}$ & $-\zeta$ & $\zeta e_{1}$ \\
$e_{3}$ & $e_{3}$ & $\sigma e_{2}$ & $-\zeta e_{1}$ & $-\sigma \zeta$
\end{tabular}

$(\sigma, \zeta)=(1,1)$ için $K$, Hamilton kuaterniyonlarını verirken, $(\sigma, \zeta)=(1,-1)$ için ise split - kuaterniyonları verir. Buna göre, $i=0,1,2,3$ için $a_{i}$ ve $b_{i} \in \mathbb{R}$ olmak üzere $q_{1}=a_{0}+a_{1} e_{1}+a_{2} e_{2}+a_{3} e_{3} \quad$ ve $q_{2}=b_{0}+b_{1} e_{1}+b_{2} e_{2}+b_{3} e_{3}$ iki genelleştirilmiş kuaterniyon ise toplama işlemi

$q_{1}+q_{2}=\left(a_{0}+b_{0}\right)+\left(a_{1}+b_{1}\right) e_{1}+\left(a_{2}+b_{2}\right) e_{2}+\left(a_{3}+b_{3}\right) e_{3}$,

çarpma işlemi ise

$$
\begin{aligned}
q_{1} q_{2}=a_{0} b_{0}-\sigma a_{1} b_{1}- & \zeta a_{2} b_{2}-\sigma \zeta a_{3} b_{3}+\left(a_{0} b_{1}+a_{1} b_{0}+\zeta a_{2} b_{3}-\zeta a_{3} b_{2}\right) e_{1} \\
& +\left(a_{0} b_{2}-\sigma a_{1} b_{3}+a_{2} b_{0}+\sigma a_{3} b_{1}\right) e_{2}+\left(a_{0} b_{3}+a_{1} b_{2}-a_{2} b_{1}+a_{3} b_{0}\right) e_{3}
\end{aligned}
$$

şeklinde tanımlanır. $q_{1}$ katerniyonunun eşleniği

$\overline{q_{1}}=a_{0}-a_{1} e_{1}-a_{2} e_{2}-a_{3} e_{3}$

iken normu ise 


$$
N\left(\overline{q_{1}}\right)=a_{0}^{2}+a_{1}^{2} \sigma+a_{2}^{2} \zeta+a_{3}^{2} \sigma \zeta
$$

şeklindedir.

Fibonacci kuaterniyonları Horadam (1963) ve Lucas kuaterniyonları ise Iyer (1969) tarafindan

$$
Q_{n}=F_{n}+F_{n+1} e_{1}+F_{n+2} e_{2}+F_{n+3} e_{3} \text { ve } K_{n}=L_{n}+L_{n+1} e_{1}+L_{n+2} e_{2}+L_{n+3} e_{3}
$$

olarak tanımlanmıştır. Halıcı (2012) Fibonacci ve Lucas kuaterniyonları için Binet formüllerini

$$
Q_{n}=\frac{\alpha \alpha^{n}-\beta \beta^{n}}{\alpha-\beta} \text { ve } K_{n}=\alpha \alpha^{n}+\beta \beta^{n}
$$

şeklinde vermiş ve bu formülleri kullanarak bazı özdeşlikler hesaplamıştır. Akyiğit vd. (2013), split Fibonacci kuaterniyonlarını incelemişlerdir. Sonrasında Akyiğit vd. (2014), genelleştirilmiş Fibonacci ve Lucas kuaterniyonlarını çalışmışlardır. Ramirez (2015), $k$ - Fibonacci ve $k$-Lucas kuaterniyonlarını tanımladıktan sonra Polatlı vd. (2016), split $k$ - Fibonacci ve $k$-Lucas kuaterniyonlarını incelemişlerdir. Bilgici vd. (2017) ise $k$ - Fibonacci ve $k$ - Lucas genelleştirilmiş kuaterniyonlarını tanımlayarak bu sayılar için birçok özdeşlik vermişlerdir.

Ek olarak Pell ve Pell - Lucas kuaterniyonları hakkında benzer birçok çalışma göze çarpmaktadır (Çimen ve İpek, 2016; Catarino, 2016; Tokeşer vd., 2017).

Yukarıda bahsedilen çalışmaların tamamında $\left\{1, e_{1}, e_{2}, e_{3}\right\}$ tabanının katsayıları, (3) eşitliğine benzer şekilde ardışık Fibonacci, Lucas, Pell veya Pell - Lucas sayıları olarak seçilmiştir. Benzer durum Bilgici vd. (2017)'nin $k$ - Fibonacci ve $k$ - Lucas genelleştirilmiş kuaterniyonları tanımında da görülmektedir. Bu çalışmanın yukarıda zikredilenlerden farkı, $\left\{1, e_{1}, e_{2}, e_{3}\right\}$ tabanının katsayılarının rasgele $k$ - Fibonacci ve $k$ - Lucas sayısı olarak atanabilmesidir. Buna göre kısıtlamasız $k$ - Fibonacci ve $k$-Lucas genelleştirilmiş kuaterniyonları aşă̆ıdaki gibi tanımlanır.

\section{Tanim 1.1:}

$k$ bir reel sayı, $n$ negatif olmayan bir tamsayı, $a, b$ ve $c$ tamsayılar olmak üzere, kısıtlamasız $k-$ Fibonacci ve $k$ - Lucas genelleştirilmiş kuaterniyonları sırasıyla;

$$
Y_{k, n}^{(a, b, c)}=F_{k, n}+F_{k, n+a} e_{1}+F_{k, n+b} e_{2}+F_{k, n+c} e_{3}
$$

ve

$$
Z_{k, n}^{(a, b, c)}=L_{k, n}+L_{k, n+a} e_{1}+L_{k, n+b} e_{2}+L_{k, n+c} e_{3}
$$

şeklinde tanımlanır.

$$
\begin{aligned}
& F_{k,-n}=(-1)^{n+1} F_{k, n} \text { ve } L_{k,-n}=(-1)^{n} L_{k, n} \text { bağıntıları ile (4) ve (5) eşitlikleri vasıtasıyla } \\
& Y_{k,-n}^{(a, b, c)}=(-1)^{n+1}\left[F_{k, n}+(-1)^{a+1} F_{n-a}+(-1)^{b+1} F_{n-b}+(-1)^{c+1} F_{n-c}\right] \\
& \text { ve } \\
& Z_{k,-n}^{(a, b, c)}=(-1)^{n}\left[L_{k, n}+(-1)^{a} L_{n-a}+(-1)^{b} L_{n-b}+(-1)^{c} L_{n-c}\right]
\end{aligned}
$$

bulunur. Ayrıca $k$ - Fibonacci ve $k$ - Lucas sayılarının tanımından kolayca 


$$
Y_{k, n}^{(a, b, c)}=k Y_{k, n-1}^{(a, b, c)}+Y_{k, n-2}^{(a, b, c)} \text { ve } Z_{k, n}^{(a, b, c)}=k Z_{k, n-1}^{(a, b, c)}+Z_{k, n-2}^{(a, b, c)}
$$

eşitliği elde edilir.

\section{2. Üreteç fonksiyonları ve binet formülleri}

Kısıtlamasız $k$ - Fibonacci $\left\{Y_{k, n}^{(a, b, c)}\right\}$ ve $k$ - Lucas $\left\{Z_{k, n}^{(a, b, c)}\right\}$ genelleştirilmiş kuaterniyon dizileri için üreteç fonksiyonları aşağıdaki teoremde verilmiştir.

\section{Teorem 2.1:}

$k$ bir reel sayı, $a, b$ ve $c$ tamsayılar olmak üzere $\left\{Y_{k, n}^{(a, b, c)}\right\}$ ve $\left\{Z_{k, n}^{(a, b, c)}\right\}$ dizilerinin üreteç fonksiyonları

$$
\sum_{n=0}^{\infty} Y_{k, n}^{(a, b, c)} x^{n}=\frac{Y_{k, 0}^{(a, b, c)}+x\left(Y_{k, 1}^{(a, b, c)}-k Y_{k, 0}^{(a, b, c)}\right)}{1-k x-x^{2}}
$$

ve

$$
\sum_{n=0}^{\infty} Z_{k, n}^{(a, b, c)} x^{n}=\frac{Z_{k, 0}^{(a, b, c)}+x\left(Z_{k, 1}^{(a, b, c)}-k Z_{k, 0}^{(a, b, c)}\right)}{1-k x-x^{2}}
$$

şeklindedir.

Ispat: $F(x)=\sum_{n=0}^{\infty} Y_{k, n}^{(a, b, c)} x^{n}$ olsun. $F(x)$, sirasıyla $1,-k x$ ve $x^{2}$ ile çarpılırsa

$$
\begin{aligned}
& F(x)=Y_{k, 0}^{(a, b, c)}+Y_{k, 1}^{(a, b, c)} x+\sum_{n=2}^{\infty} Y_{k, n}^{(a, b, c)} x^{n}, \\
& -k x F(x)=-k Y_{k, 0}^{(a, b, c)} x+\sum_{n=2}^{\infty} Y_{k, n-1}^{(a, b, c)} x^{n}
\end{aligned}
$$

ve

$$
-x^{2} F(x)=-\sum_{n=2}^{\infty} Y_{k, n-2}^{(a, b, c)} x^{n}
$$

elde edilir. (9), (10) ve (11) eşitlikleri taraf tarafa toplanıp (6) denklemi kullanılırsa

$$
\left(1-k x-x^{2}\right) F(x)=Y_{k, 0}^{(a, b, c)}+x\left(Y_{k, 1}^{(a, b, c)}-k Y_{k, 0}^{(a, b, c)}\right)
$$

bulunur ki, bu ise (7) eşitliğini verir. (8) eşitliğinin ispatı benzer şekilde yapılır. 口

Kısıtlamasız $k$-Fibonacci ve $k$-Lucas genelleştirilmiş kuaterniyonları için Binet formülleri aşağıdaki teoremde verilmiştir.

\section{Teorem 2.2:}

$k$ bir reel sayı, $n, a, b$ ve $c$ tamsayılar olmak üzere $n$-yinci kısıtlamasız $k$-Fibonacci ve $k$ - Lucas genelleştirilmiş kuaterniyonları sırasıyla

$$
Y_{k, n}^{(a, b)}=\frac{\chi \chi^{n}-\mu \mu^{n}}{\chi-\mu}
$$

ve

$$
Z_{k, n}^{(a, b, c)}=\chi \chi^{n}+\mu \mu^{n}
$$


şeklindedir. Burada

$$
\chi=1+\chi^{a} e_{1}+\chi^{b} e_{2}+\chi^{c} e_{3}
$$

ve

$$
\mu=1+\mu^{a} e_{1}+\mu^{b} e_{2}+\mu^{c} e_{3}
$$

dir.

İspat: (1) Binet formülü ve (4) eşitliği kullanarak

$$
\begin{aligned}
Y_{k, n}^{(a, b, c)}= & F_{k, n}+F_{k, n+a} e_{1}+F_{k, n+b} e_{2}+F_{k, n+c} e_{3} \\
& =\frac{1}{\chi-\mu}\left[\left(\chi^{n}-\mu^{n}\right)+\left(\chi^{n+a}-\mu^{n+a}\right) e_{1}+\left(\chi^{n+b}-\mu^{n+b}\right) e_{2}+\left(\chi^{n+c}-\mu^{n+c}\right) e_{3}\right] \\
& =\frac{1}{\chi-\mu}\left[\left(\chi^{n}+\chi^{n+a} e_{1}+\chi^{n+b} e_{2}+\chi^{n+c} e_{3}\right)-\left(\mu^{n}+\mu^{n+a} e_{1}+\mu^{n+b} e_{2}+\mu^{n+c} e_{3}\right)\right] \\
& =\frac{1}{\chi-\mu}\left[\left(1+\chi^{a} e_{1}+\chi^{b} e_{2}+\chi^{c} e_{3}\right) \chi^{n}-\left(1+\mu^{a} e_{1}+\mu^{b} e_{2}+\mu^{c} e_{3}\right) \mu^{n}\right]
\end{aligned}
$$

bulunur. Son eşitlik denklem (12)'yi ispatlarken, denklem (13)'ün ispatı benzer şekilde yapılır. Aşağıdaki sonuç ileride elde edilecek olan özdeşliklerin ispatlarında kullanılacaktır.

\section{Lemma 2.3:}

$k$ bir reel sayı, $a, b$ ve $c$ tamsayılar olmak üzere

$$
\chi \mu=A+\sqrt{k^{2}+4} B
$$

ve

$$
\mu \chi=A-\sqrt{k^{2}+4} B
$$

dir. Burada

$$
A=Z_{k, 0}^{(a, b, c)}-1-\sigma(-1)^{a}-\zeta(-1)^{b}-\sigma \zeta(-1)^{c}
$$

ve

$$
B=\zeta(-1)^{c} F_{k, b-c} e_{1}+\sigma(-1)^{a} F_{k, c-a} e_{2}+(-1)^{b} F_{k, a-b} e_{3}
$$

şeklindedir.

Ispat: (14) ve (15) eşitlikleri çarpılırsa

$$
\begin{aligned}
& \chi \mu=\left(1+\chi^{a} e_{1}+\chi^{b} e_{2}+\chi^{c} e_{3}\right)\left(1+\mu^{a} e_{1}+\mu^{b} e_{2}+\mu^{c} e_{3}\right) \\
&=1-\sigma(-1)^{a}-\zeta(-1)^{b}-\sigma \zeta(-1)^{c}+\left(\chi^{a}+\mu^{a}+\zeta \chi^{b} \mu^{c}-\zeta \chi^{c} \mu^{b}\right) e_{1} \\
&+\left(\chi^{b}+\mu^{b}+\sigma \chi^{c} \mu^{a}-\sigma \chi^{a} \mu^{c}\right) e_{2}+\left(\chi^{c}+\mu^{c}+\chi^{a} \mu^{b}-\chi^{b} \mu^{a}\right) e_{3} \\
&=1-\sigma(-1)^{a}-\zeta(-1)^{b}-\sigma \zeta(-1)^{c}+\left[L_{k, a}+\zeta(-1)^{c}\left(\chi^{b-c}-\mu^{b-c}\right)\right] e_{1} \\
&+\left[L_{k, b}+\sigma(-1)^{a}\left(\chi^{c-a}-\mu^{c-a}\right)\right] e_{2}+\left[L_{k, c}+(-1)^{b}\left(\chi^{a-b}-\mu^{a-b}\right)\right] e_{3} \\
&=Z_{k, 0}^{(a, b, c)}-1-\sigma(-1)^{a}-\zeta(-1)^{b}-\sigma \zeta(-1)^{c}
\end{aligned}
$$




$$
+\sqrt{k^{2}+4}\left[\zeta(-1)^{c} F_{k, b-c} e_{1}+\sigma(-1)^{a} F_{k, c-a} e_{2}+(-1)^{b} F_{k, a-b} e_{3}\right]
$$

elde edilir. Son eşitlik denklem (16)'yı verir. Denklem (17) benzer şekilde elde edilir. 口

\section{Sonuçlar}

$\mathrm{Bu}$ bölümde yukarıdaki bilgilerin 1şığı altında kısıtlamasız $k$-Fibonacci ve $k$-Lucas genelleştirilmiş kuaterniyonlarının bazı özellikleri elde edilecektir. İlk olarak en genel özdeşliklerden birisi olan Vajda özdeşliği aşă̆ıdaki teoremde verilmiştir.

\section{Teorem 3.1:}

Her $k$ reel sayısı, $r, s, t, a, b$ ve $c$ tamsayıları için

$$
\begin{aligned}
& Y_{k, r+s}^{(a, b, c)} Y_{k, r+t}^{(a, b, c)}-Y_{k, r}^{(a, b, c)} Y_{k, r+s+t}^{(a, b, c)}=(-1)^{r} F_{k, s}\left(A F_{k, t}-B L_{k, t}\right) \\
& \text { ve } \\
& \quad Z_{k, r+s}^{(a, b, c)} Z_{k, r+t}^{(a, b, c)}-Z_{k, r}^{(a, b, c)} Z_{k, r+s+t}^{(a, b, c)}=(-1)^{r+1}\left(k^{2}+4\right) F_{k, s}\left(A F_{k, t}-B L_{k, t}\right)
\end{aligned}
$$

dir.

Ispat: Kısıtlamasız $k$ - Fibonacci genelleştirilmiş kuaterniyonlarının Binet formülünden

$$
\begin{aligned}
Y_{k, r+s}^{(a, b, c)} Y_{k, r+t}^{(a, b, c)}-Y_{k, r}^{(a, b, c)} Y_{k, r+s+t}^{(a, b, c)} \\
=\frac{1}{k^{2}+4}\left[\left(\chi \chi^{r+s}-\mu \mu^{r+s}\right)\left(\chi \chi^{r+t}-\mu \mu^{r+t}\right)-\left(\chi \chi^{r}-\mu \mu^{r}\right)\left(\chi \chi^{r+s+t}-\mu \mu^{r+s+t}\right)\right] \\
=\frac{1}{k^{2}+4}\left(-\chi \mu \chi^{r+s} \mu^{r+t}+\chi \mu \chi^{r} \mu^{r+s+t}-\mu \chi \chi^{r+t} \mu^{r+s}+\mu \chi \chi^{r+s+t} \mu^{r}\right) \\
=\frac{(-1)^{r}}{k^{2}+4}\left(-\chi \mu \chi^{s} \mu^{t}+\chi \mu \mu^{s+t}-\mu \chi \chi^{t} \mu^{s}+\mu \chi \chi^{s+t}\right) \\
=\frac{(-1)^{r}}{k^{2}+4}\left[-\chi \mu \mu^{t}\left(\chi^{s}-\mu^{s}\right)+\mu \chi \chi^{t}\left(\chi^{s}-\mu^{s}\right)\right] \\
=\frac{(-1)^{r+1} F_{k, s}}{\sqrt{k^{2}+4}}\left[\chi \mu \mu^{t}-\mu \chi \chi^{t}\right] \\
=\frac{(-1)^{r+1} F_{k, s}}{\sqrt{k^{2}+4}}\left[\left(A+\sqrt{k^{2}+4} B\right) \mu^{t}-\left(A-\sqrt{k^{2}+4} B\right) \chi^{t}\right] \\
=(-1)^{r} F_{k, s}\left(A F_{k, t}-B L_{k, t}\right)
\end{aligned}
$$

elde edilir. Son eşitlik denklem (18)'i ispatlar. Denklem (19) benzer şekilde elde edilir. 口

Vajda özdeşliğini kullanarak Catalan ve Cassini özdeşlikleri elde edilebilir. Vajda özdeşliğinde $t \rightarrow-s$ alınırsa aşağıdaki teoremde verilen Catalan özdeşliği elde edilir.

\section{Teorem 3.2.}

Her $k$ reel sayıs1, $r, s, a, b$ ve $c$ tamsayıları için

$$
Y_{k, r+s}^{(a, b, c)} Y_{k, r-s}^{(a, b, c)}-\left[Y_{k, r}^{(a, b, c)}\right]^{2}=(-1)^{r+s+1}\left(A F_{k, s}^{2}+B F_{k, s} L_{k, s}\right)
$$

ve

$$
Z_{k, r+s}^{(a, b, c)} Z_{k, r-s}^{(a, b, c)}-\left[Z_{k, r}^{(a, b, c)}\right]^{2}=(-1)^{r+s}\left(k^{2}+4\right)\left(A F_{k, s}^{2}+B F_{k, s} L_{k, s}\right)
$$


dir.

Catalan özdeşliğinde $s \rightarrow 1$ alınırsa Cassini özdeşliği aşağıdaki gibi bulunur.

\section{Teorem 3.3.}

Her $k$ bir reel sayısı, $r, a, b$ ve $c$ tamsayıları için

$$
\begin{aligned}
& Y_{k, r+1}^{(a, b, c)} Y_{k, r-1}^{(a, b, c)}-\left[Y_{k, r}^{(a, b, c)}\right]^{2}=(-1)^{r}(A+k B) \\
& \mathrm{ve} \\
& Z_{k, r+1}^{(a, b, c)} Z_{k, r-1}^{(a, b, c)}-\left[Z_{k, r}^{(a, b, c)}\right]^{2}=(-1)^{r+1}\left(k^{2}(A+k B)\right.
\end{aligned}
$$

dir.

Bir başka önemli özdeşlik aşağıdaki teoremde verilen d'Ocagne özdeşliğidir.

\section{Teorem 3.4.}

Her $k$ reel sayısı, $r, s, a, b$ ve $c$ tamsayıları için

$$
Y_{k, r}^{(a, b, c)} Y_{k, s+1}^{(a, b, c)}-Y_{k, r+1}^{(a, b, c)} Y_{k, s}^{(a, b, c)}=(-1)^{s}\left[A F_{k, r-s}+B L_{r-s}\right]
$$

ve

$$
Z_{k, r}^{(a, b, c)} Z_{k, s+1}^{(a, b, c)}-Z_{k, r+1}^{(a, b, c)} Z_{k, s}^{(a, b, c)}=(-1)^{s+1}\left(k^{2}+4\right)\left[A F_{k, r-s}+B L_{r-s}\right]
$$

dir.

Ispat: Kısıtlamasız $k$ - Lucas genelleştirilmiş kuaterniyonlarının Binet formülünden

$$
\begin{aligned}
Z_{k, r}^{(a, b)} Z_{k, s+1}^{(a, b, c)}-Z_{k, r+1}^{(a, b, c)} Z_{k, s}^{(a, b, c)} \\
\quad=\left(\chi \chi^{r}+\mu \mu^{r}\right)\left(\chi \chi^{s+1}+\mu \mu^{s+1}\right)-\left(\chi \chi^{r+1}+\mu \mu^{r+1}\right)\left(\chi \chi^{s}+\mu \mu^{s}\right) \\
\quad=\chi \mu \chi^{r} \mu^{s+1}-\chi \mu \chi^{r+1} \mu^{s}+\mu \chi \chi^{s+1} \mu^{r}-\mu \chi \chi^{s} \mu^{r+1} \\
\quad=(-1)^{s}\left[-\chi \mu \chi^{r-s}(\chi-\mu)+\mu \chi \mu^{r-s}(\chi-\mu)\right] \\
\quad=(-1)^{s+1}\left(\sqrt{k^{2}+4}\right)\left[\chi \mu \chi^{r-s}-\mu \chi \mu^{r-s}\right] \\
=(-1)^{s+1}\left(\sqrt{k^{2}+4}\right)\left[\left(A+\sqrt{k^{2}+4} B\right) \chi^{r-s}-\left(A-\sqrt{k^{2}+4} B\right) \mu^{r-s}\right] \\
\quad=(-1)^{s+1}\left(\sqrt{k^{2}+4}\right)\left[A\left(\chi^{r-s}-\mu^{r-s}\right)+\sqrt{k^{2}+4} B\left(\chi^{r-s}+\mu^{r-s}\right)\right]
\end{aligned}
$$

bulunur ki, son eşitlik denklem (25)'i verir. Denklem (24) benzer şekilde elde edilir. 口 Son olarak aşağıdaki özdeşlikler verilecektir.

\section{Teorem 3.5.}

Her $k$ bir reel sayısı, $r, s, a, b$ ve $c$ tamsayıları için

$$
\begin{aligned}
& Z_{k, r}^{(a, b, c)}=Y_{k, r-1}^{(a, b, c)}+Y_{k, r+1}^{(a, b, c)} \\
& Z_{k, r}^{(a, b, c)} Y_{k, r-1}^{(a, b, c)}=Y_{k, 2 r}^{(a, b, c)}-F_{k, 2 r}-\sigma F_{k, 2 r+2 a}-\zeta F_{k, 2 r+2 b}-\sigma \zeta F_{k, 2 r+2 c}+B(-1)^{r},
\end{aligned}
$$




$$
\begin{gathered}
\sum_{r=1}^{s} Y_{k, r}^{(a, b, c)}=\frac{1}{k}\left[Y_{k, r+1}^{(a, b, c)}+Y_{k, r}^{(a, b, c)}-\left(1+e_{1}+e_{2}+e_{3}\right)\right], \\
\sum_{r=1}^{s} Z_{k, r}^{(a, b, c)}=\frac{1}{k}\left[Z_{k, r+1}^{(a, b, c)}+Z_{k, r}^{(a, b, c)}\right]+\left(1-\frac{2}{k}\right)\left(1+e_{1}+e_{2}+e_{3}\right) .
\end{gathered}
$$

dir.

İspat: Her $r$ tamsayısı için, $k$ - Fibonacci ve $k$ - Lucas sayıları arasında

$$
L_{k, r}=F_{k, r-1}+F_{k, r+1}
$$

özdeşliği bulunmaktadır (Falcon, 2011). Dolayısıyla her $r, a, b$ ve $c$ tamsayıları için

$$
\begin{aligned}
Y_{k, r-1}^{(a, b, c)}+Y_{k, r+1}^{(a, b, c)} & =\left(F_{k, r-1}+F_{k, r+a-1} e_{1}+F_{k, r+b-1} e_{2}+F_{k, r+c-1} e_{3}\right)+\left(F_{k, r+1}+F_{k, r+a+1} e_{1}+F_{k, r+b+1} e_{2}+F_{k, r+c+1} e_{3}\right) \\
& =\left(F_{k, r-1}+F_{k, r+1}\right)+\left(F_{k, r+a-1}+F_{k, r+a+1}\right) e_{1}+\left(F_{k, r+b-1}+F_{k, r+b+1}\right) e_{2}+\left(F_{k, r+c-1}+F_{k, r+c+1}\right) e_{3} \\
& =L_{k, r}+L_{k, r+a} e_{1}+L_{k, r+b} e_{2}+L_{k, r+c} e_{3} \\
& =Z_{k, r}^{(a, b, c)}
\end{aligned}
$$

elde edilir ki, bu ise denklem (26)'yı ispatlar. Falcon ve Plaza (2007), $k$ - Fibonacci sayılarının ilk $s$ teriminin toplamı için

$$
\sum_{r=1}^{s} F_{k, r}=\frac{1}{k}\left(F_{k, s+1}+F_{k, s}-1\right)
$$

özdeşliğini vermişlerdir. Bu doğrultuda

$$
\begin{aligned}
\sum_{r=1}^{s} Y_{k, r}^{(a, b, c)}= & \sum_{r=1}^{s}\left(F_{k, r}+F_{k, r+a} e_{1}+F_{k, r+b} e_{2}+F_{k, r+c} e_{3}\right) \\
= & \sum_{r=1}^{s} F_{k, r}+e_{1} \sum_{r=1}^{s} F_{k, r+a}+e_{2} \sum_{r=1}^{s} F_{k, r+b}+e_{3} \sum_{r=1}^{s} F_{k, r+c} \\
= & \frac{1}{k}\left[F_{k, r+1}+F_{k, r}-1+e_{1}\left(F_{k, r+a+1}+F_{k, r+a}-1\right)\right. \\
& \left.\quad+e_{2}\left(F_{k, r+b+1}+F_{k, r+b}-1\right)+e_{3}\left(F_{k, r+c+1}+F_{k, r+c}-1\right)\right] \\
= & \frac{1}{k}\left[\left(F_{k, r+1}+F_{k, r+1+a} e_{1}+F_{k, r+1+b} e_{2}+F_{k, r+1+c} e_{3}\right)\right. \\
\quad & \left.\quad\left(F_{k, r}+F_{k, r+a} e_{1}+F_{k, r+b} e_{2}+F_{k, r+c} e_{3}\right)-\left(1+e_{1}+e_{2}+e_{3}\right)\right] \\
= & \frac{1}{k}\left[Y_{k, r+1}^{(a, b, c)}+Y_{k, r}^{(a, b, c)}-\left(1+e_{1}+e_{2}+e_{3}\right)\right]
\end{aligned}
$$

elde edilir ki, denklem (28) ispatlanmış olur. Benzer şekilde Falcon (2011)'un vermiş olduğu $\sum_{r=1}^{s} L_{k, r}=1+\frac{1}{k}\left(L_{k, s+1}+L_{k, s}-2\right)$

özdeşliği kullanılarak denklem (29) ispatlanabilir. 


\section{Kaynaklar}

Akyigit, M., Kosal, H.H. and Tosun, M. (2013). Split Fibonacci quaternions. Advances in Applied Clifford Algebras, 23(3), 535-545, https://doi.org/10.1007/s00006-013-0401-9

Akyigit, M., Kosal, H.H. and Tosun, M. (2014). Fibonacci generalized quaternions. Advances in Applied Clifford Algebras, 24(3), 631-641, https://doi.org/10.1007/s00006-014-0458-0

Bilgici, G., Tokeser, U. and Unal, Z. (2017). kFibonacci and k-Lucas generalized quaternions. Konuralp Journal of Mathematics, 5(2), 102 113.

Catarino, P. (2016). The modified Pell and the modified k-Pell quaternions and octonions. Advances in Applied Clifford Algebras, 26(2), 577-590, https://doi.org.10.1007/s00006-015-0611-4

Çimen, C.B. and İpek, A. (2016). On Pell quaternions and Pell-Lucas quaternions. Advances in Applied Clifford Algebras, 26(1), 39-51, https://doi.org/10.1007/s00006-015-0571-8

Falcon, S. and Plaza, A. (2007). The k-Fibonacci sequence and the Pascal 2-triangle. Chaos Solitons Fractals, 33(1), 38-49, https://doi.org/10.1016/j.chaos.2006.10.022

Falcon, S. (2011). On the k-Lucas numbers. International Journal of Contemporary Mathematical Sciences, 21, 1039-1050.
Halıc1, S. (2012). On Fibonacci quaternions. Advances in Applied Clifford Algebras, 22(2), 321-327, https://doi.org/10.1007/s00006-011-0317-1

Hamilton, W.R. (1853). Lectures on quaternions. Dublin: Hodges and Smith.

Horadam, A. F. (1963). Complex Fibonacci numbers and Fibonacci quaternions. The American Mathematical Monthly, 70, 289-291.

Iyer, M. R. (1969). Some result on Fibonacci quaternions. Fibonacci Quarterly, 7(2), 201-210.

Koshy, T. (2001). Fibonacci and Lucas numbers with applications. Canada: A Wiley-Interscience Publication.

Koshy, T. (2014). Pell and Pell-Lucas numbers with applications. New York: Springer-Verlag.

Polatl1, E. and Kesim, S. (2015). A Note on Catalan's identity for the k- Fibonacci quaternions. Journal of Integer Sequence, 18, 1-4.

Ramirez, J. L. (2015). Some combinatorial properties of the k-Fibonacci and the k-Lucas quaternions. Analele Stiintifice ale Universitatii Ovidius Constanta Seria Matematica, 23(2), 201-212, https://doi.org/10.1515/auom-2015-0037

Tokeser, U., Unal, Z. and Bilgici, G. (2017). Split Pell and split Pell-Lucas quaternions. Advances in Applied Clifford Algebras, 27(2), 1881-1893, https://doi.org/10.1007/s00006-016-0747-x 